\title{
Postinfectious Anti-Myelin Oligodendrocyte Glycoprotein Antibody Positive Optic Neuritis and Myelitis
}

\author{
J. P. Vieira, MD', J. Sequeira, MD², and M. J. Brito, $M D^{3}$
}

\begin{abstract}
We report the case of a 9-year-old girl admitted with fever, headache, and a cerebrospinal fluid lymphocytic pleocytosis. Polymerase chain reaction was positive for human herpes virus 6 . She subsequently developed ataxia and bilateral loss of vision. Magnetic resonance imaging (MRI) showed bilateral optic nerve lesions with extension to optic chiasm and a short-segment myelitis. Serologic studies were positive for Borrelia burgdorferi IgM. Anti-aquaporin 4 antibody was negative and anti-myelin oligodendrocyte glycoprotein antibody (MOG) positive. After intravenous methylprednisolone, ceftriaxone, and intravenous immunoglobulin, her vision slowly recovered. The patient was discharged with only mild visual acuity loss, I month after admission. Brain MRI was repeated later and was normal and MOG assay became negative. In our view, this patient suffered from a postinfectious, anti-MOG-mediated, spinal cord and optic nerve demyelination.
\end{abstract}

\section{Keywords}

myelin-oligodendrocyte glycoprotein antibodies, bilateral optic neuritis, neuromyelitis optica spectrum disorders, Lyme borreliosis

Received December 26, 2016. Received revised June 24, 2017. Accepted for publication July 5, 2017.

Borrelia burgdorferi infection is associated with neurologic manifestations in up to $15 \%$ of cases. ${ }^{1}$ Optic neuritis related with Lyme borreliosis has been reported infrequently and there is uncertainty about the validity of the diagnosis in many cases. ${ }^{2}$ Neurologic manifestations associated with human herpes virus 6 (HHV6) infection are usually febrile seizures in infants and encephalitis in immunosuppressed patients. ${ }^{3}$ Optic neuritis may have multiple causes, including infection and several autoimmune diseases, such as multiple sclerosis, acute disseminated encephalomyelitis, or neuromyelitis optica spectrum disorders.

We describe a case of one female adolescent with antimyelin oligodendrocyte glycoprotein (MOG)-positive optic neuritis and myelitis. She had a positive polymerase chain reaction for HHV6 in cerebrospinal fluid and positive enzyme-linked immunosorbent assay and Western blot for Borrelia burgdorferi in serum. We propose that our case expands the spectrum of the association between infection and immunemediated MOG demyelination.

\section{Case Report}

A previously healthy 9-year-old girl of African descent living in the Azores Islands was admitted to her local hospital with a 6-day history of a febrile illness, headache, and somnolence, previously treated with amoxicillin-clavulanic acid without resolution of symptoms. She had no history of recent travel. Her white blood cell count was $16340 / \mu \mathrm{L}$ ( $80 \%$ neutrophils) and c-reactive protein $0.88 \mathrm{mg} / \mathrm{dL}$. Cerebrospinal fluid examination revealed a cell count of 95 cells $/ \mu \mathrm{L}$, with lymphocytic predominance and elevated protein $(66 \mathrm{mg} / \mathrm{dL})$. Polymerase chain reaction in the cerebrospinal fluid was positive for HHV6 and negative for Borrelia burgdorferi, and no serologic tests were performed regarding HHV6. Four days later, she presented with gait imbalance, abdominal pain, and dysuria. Neurologic examination was positive for gait ataxia with a positive Romberg sign. She underwent treatment with a 21-day course of ganciclovir $(10 \mathrm{mg} / \mathrm{kg} / \mathrm{d})$ and was discharged on the 26th day after admission with a normal physical examination. Brain

\footnotetext{
'Neurology Department, Hospital Dona Estefânia, Centro Hospitalar de Lisboa Central, Lisbon, Portugal

${ }^{2}$ Neurology Department, Centro Hospitalar de Lisboa Central, Lisbon, Portugal

${ }^{3}$ Infectious Diseases Unit, Hospital Dona Estefânia, Centro Hospitalar de Lisboa Central, Lisbon, Portugal
}

\section{Corresponding Author:}

J. P. Vieira, MD, Neurology Department, Hospital Dona Estefânia, Centro Hospitalar de Lisboa Central, Rua Jacinta Marto, I 169-045 Lisbon, Portugal.

Email: jose.vieira@chlc.min-saude.pt 


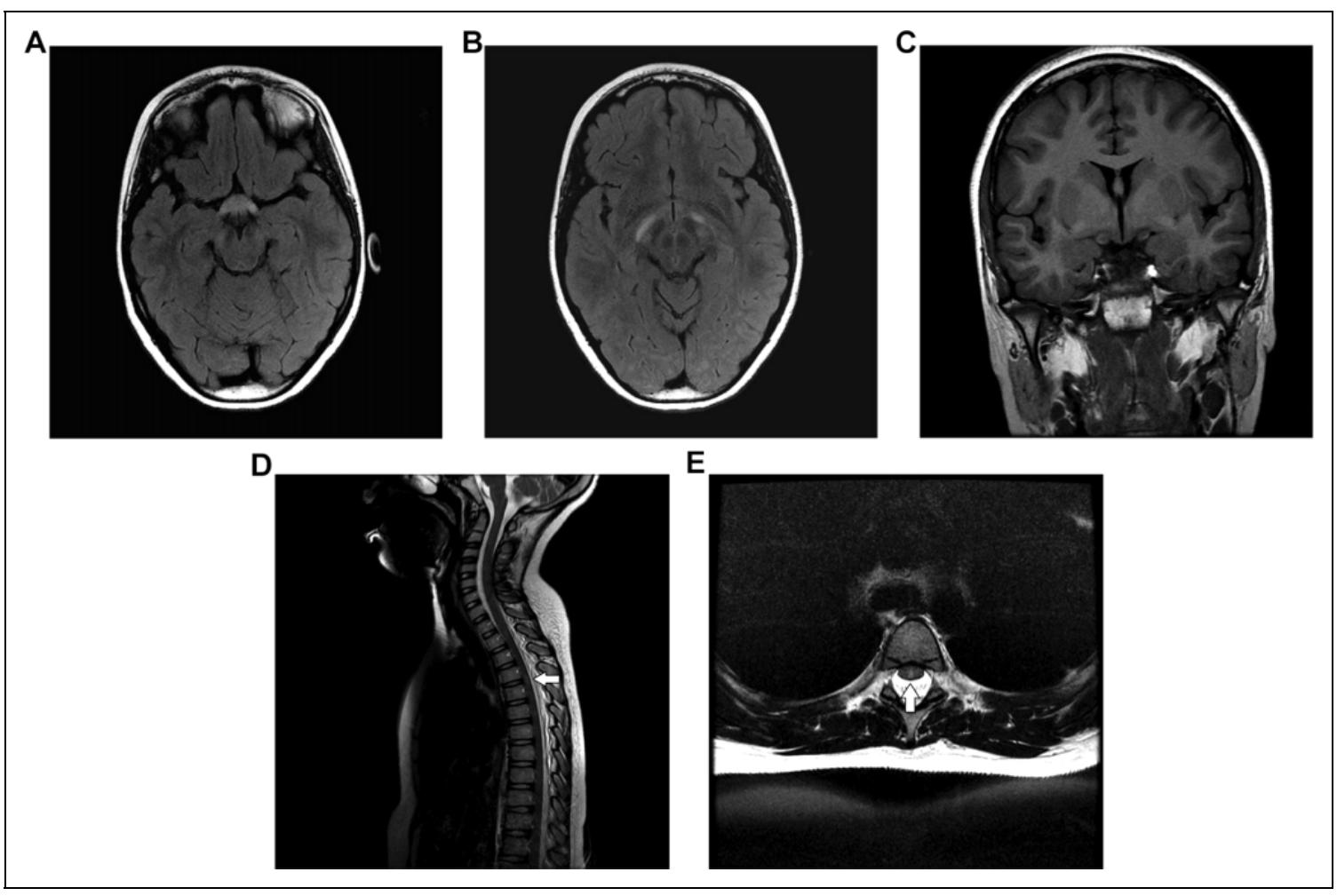

Figure I. Fluid-attenuated inversion recovery T2 hypersignal and edema in the optic nerves and optic chiasm (A), optic tracts and lateral geniculate bodies $(B)$ and $(C)$, and in the dorsal spinal cord (D and $E)$.

magnetic resonance imaging (MRI) was normal. She was readmitted 11 days later with acute, painless, and bilateral but asymmetric reduction of visual acuity.

The patient was then referred to our hospital for further investigation. Visual acuity was severely impaired (visual acuity 20/200 right eye, 20/100 left eye); a left-sided afferent pupillary defect was present and there was bilateral optic disc edema and ataxia. Considering that the present symptoms and signs were delayed more than 1 month after the early febrile disease, we admitted that an inflammatory mechanism could underlie this clinical presentation and began treatment with intravenous methylprednisolone ( $30 \mathrm{mg} / \mathrm{kg} / \mathrm{d}$ for 5 days) with a progressive but slow recovery of vision.

Brain and spinal cord MRI showed a T2 and T2 fluidattenuated inversion recovery hypersignal involving the posterior optic nerves and optic chiasm, optic tracts, lateral geniculate nuclei, and 3 small lesions in the posterior columns of the dorsal spinal cord at T2, T4-T5, and T6 levels (Figure 1). There was no contrast enhancement.

Visual evoked potentials showed P100 waves with reduced amplitude (pattern VEPs: P100 amplitude $4.5 \mu \mathrm{V}$ right eye, 2.7 $\mu \mathrm{V}$ left eye) and prolonged latency (pattern VEPs: P100 latency $154 \mathrm{~ms}$ right eye, $196 \mathrm{~ms}$ left eye).

Routine blood testing revealed a white blood cell count of $14800 / \mu \mathrm{L}$ (neutrophils 87\%) and a gamma-glutamyl transferase of $44 \mathrm{U} / \mathrm{L}$. Immunologic workup was normal for ANA, dsDNA, ANCA, SSA, SSB, anticardiolipin antibodies, and angiotensin-converting enzyme and complement levels.
Borrelia burgdorferi IgM antibodies were positive with enzyme-linked immunosorbent assay in 2 consecutive samples, and the diagnosis of Lyme borreliosis was confirmed with immunoblot testing. Serologic tests were negative for HIV, Venereal Disease Research Laboratory, cytomegalovirus, Epstein Barr virus, Mycoplasma pneumoniae, Chlamydia pneumoniae, Toxoplasma gondii, Bartonella, Coxiella burnetii, adenovirus, and influenza $\mathrm{A} / \mathrm{B}$ antibodies.

A lumbar puncture was performed, which revealed a cerebrospinal fluid cell count of 8 cells $/ \mu \mathrm{L}$, lymphocytes, with normal protein and glucose, and no oligoclonal bands. Viral polymerase chain reaction studies for enterovirus, herpes simplex, varicella zoster, herpes hominis, cytomegalovirus, and Epstein Barr virus were negative. Polymerase chain reaction for Borrelia burgdorferi was negative. Cultures for fungi and Mycobacterium tuberculosis were negative.

Indirect immunofluorescence assays using transfected fulllength MOG and Aquaporin-4 (AQP4)-expressing cells were performed, which revealed positive anti-MOG antibodies (titer 1:32) and negative anti-AQP4 antibodies (blood samples for these studies were collected before treatment).

Considering the clinical, laboratory, and imaging findings, a diagnosis of autoimmune optic neuritis and myelitis was considered.

The patient was treated with a 21-day course of ceftriaxone (4 g daily) and a tapering dose of oral prednisolone. Because of the severe visual loss at onset and the relative delay in recovery, 
intravenous immunoglobulin was administered 14 days after methylprednisolone.

She was discharged with only mild visual loss 1 month after admission and was observed subsequently for 8 months in outpatient visits remaining asymptomatic and with a normal neurologic examination. Brain MRI was repeated 6 months after discharge and was normal. MOG assay was also repeated and became negative.

\section{Discussion}

We describe one patient with bilateral optic neuritis and myelitis following Lyme borreliosis who developed anti-MOG antibodies.

Brain MRI was performed in the second week of illness and in the sixth week. We believe that the images represent the disease evolution appropriately. The first MRI was normal and the second had no lesion outside the visual pathways and the spinal cord. We considered therefore that the diagnosis of acute disseminated encephalomyelitis without specific brain parenchymal lesions would be inappropriate and optic neuritis would not be associated with a small (not longitudinally extensive) but definite spinal cord lesion, her clinical picture being more close to neuromyelitis optica spectrum disorders.

MOG positivity is not infrequent in acute disseminated encephalomyelitis, ${ }^{4}$ in pediatric optic neuritis, ${ }^{5}$ and can be found in some cases of AQP4-seronegative neuromyelitis optica spectrum disorders. ${ }^{6,7}$ In MOG-positive neuromyelitis optica spectrum disorders compared with AQP4-positive disease, the risk for incomplete recovery and for relapse appears to be lower, as apparently occurred in our case. Monophasic MOG-positive disease has also been described ${ }^{6,7}$ and is sometimes associated with disappearance of anti-MOG antibodies.

Primary infection with HHV6 occurs in infancy and can be associated with febrile seizures. HHV6 may remain latent in monocytes and macrophages so that a positive polymerase chain reaction on cerebrospinal fluid examination is not to be interpreted as active infection without additional investigation (viral load, serum IgG). ${ }^{3}$ Immune suppression may give rise to HHV6 encephalitis and myelitis, and rare cases of encephalitis in the immunocompetent host were reported. ${ }^{4}$ Optic neuritis has been reported with HHV6 infection only twice. ${ }^{8,9}$ On clinical grounds, our patient had no evidence of immunosuppression, and so positivity for HHV6 in her first cerebrospinal fluid study could have resulted from viral latency after early childhood infection. However, we cannot without additional investigations (viral load, IgG antibodies) definitely confirm or exclude active HHV6 infection.

Our patient lives in an area endemic for Lyme borreliosis, had fever and headache, and cerebrospinal fluid lymphocytic pleocytosis. Samples of serum and cerebrospinal fluid were tested with enzyme-linked immunosorbent assay for Borrelia burgdorferi and were found to be IgM positive, as confirmed with Western blot (virtually $100 \%$ specific for early Lyme borreliosis). ${ }^{10}$ Early treatment with ceftriaxone may explain her early IgM positivity without subsequent IgG response. ${ }^{10}$
The evolution of clinical signs and symptoms, the MRI findings, and MOG positivity suggest that Borrelia infection may have triggered MOG-positive bilateral optic neuritis and myelitis.

In some animal models, immunization with MOG causes features of experimental autoimmune encephalitis with dominant optic nerve and spinal cord involvement. Thereafter, an association between optic neuritis and MOG antibodies was proposed. Although there is insufficient evidence, some studies suggest that anti-MOG antibodies might be pathogenic by inducing complement-dependent cytotoxicity, ${ }^{11}$ and this could have been operative in our case.

Our patient was treated initially with immunotherapy (intravenous methylprednisolone, intravenous immunoglobulin) and later with ceftriaxone. Ataxia resolved promptly, and later she recovered almost normal vision in both eyes. She remained well for the past 8 months. MOG positivity was present only during the active phase of her disease ( 8 months later, MOG was negative). We decided therefore to withdraw prednisolone over the course of several months, watch carefully the patient's ophthalmologic and neurologic evolution, and repeat MRI and optical coherence tomography at regular intervals.

As our period of follow-up is short, we cannot admit with certainty that a relapse will not occur in the future.

\section{Author Contributions}

JPV, JS, and MJB contributed to conception of the study and acquisition and analysis of data, drafted and revised the manuscript, and approved the final draft.

\section{Declaration of Conflicting Interests}

The authors declared no potential conflicts of interest with respect to the research, authorship, and/or publication of this article.

\section{Funding}

The authors received no financial support for the research, authorship, and/or publication of this article.

\section{Ethical Approval}

This work was approved by the Institutional Review Board and Ethics Committee of the Centro Hospitalar de Lisboa Central, Lisbon (document number 438/2017).

\section{References}

1. Marques AR. Lyme neuroborreliosis. Continuum (Minneap Minn). 2015;21:1729-1744.

2. Sibony P, Halperin J, Coyle PK, Patel KJ. Reactive Lyme serology in optic neuritis. $J$ Neuroophthalmol. 2005;25:71-82.

3. De Bolle L, Naesens L, De Clercq E. Update on human herpesvirus 6 biology, clinical features, and therapy. Clin Microbiol Rev. 2005;18:217-245.

4. Baumann M, Sahin K, Lechner C, et al. Clinical and neuroradiological differences of paediatric acute disseminating encephalomyelitis with and without antibodies to the myelin 
oligodendrocyte glycoprotein. J Neurol Neurosurg Psychiatry. 2015;86:265-272.

5. Rostasy K, Mader S, Schanda K, et al. Anti-myelin oligodendrocyte glycoprotein antibodies in pediatric patients with optic neuritis. Arch Neurol. 2012;69:752-756.

6. Sepúlveda M, Armangué T, Sola-Valls N, et al. Neuromyelitis optica spectrum disorders: comparison according to the phenotype and serostatus. Neurol Neuroimmunol Neuroinflamm. 2016;3:e225.

7. Wingerchuk DM, Banwell B, Bennett JL, et al. International consensus diagnostic criteria for neuromyelitis optica spectrum disorders. Neurology. 2015;85:177-189.

8. Moschettini D, Franceschini R, Vaccaro NM, et al. Human herpesvirus-6B active infection associated with relapsing bilateral anterior optic neuritis. J Clin Virol. 2006;37: 244-247.

9. Donati D, Méchaï F, Boutolleau D, et al. Human herpesvirus 6-associated retrobulbar optic neuritis in an HIV-infected patient: response to anti-herpesvirus therapy and long-term outcome. J Med Virol. 2007;79:931-934.

10. Stanek G, Fingerle V, Hunfeld KP, et al. Lyme borreliosis: clinical case definitions for diagnosis and management in Europe. Clin Microbiol Infect. 2011;17:69-79.

11. Mader S, Gredler V, Schanda K, et al. Complement activating antibodies to myelin oligodendrocyte glycoprotein in neuromyelitis optica and related disorders. J Neuroinflamm. 2011; $8: 184$. 